\title{
Simulation study of proton-induced and iron-induced extensive air shower at the knee energies using the new Tibet experiment
}

\author{
L. M. Zhai ${ }^{* a}$, D. Chen ${ }^{a}$, J. Huang ${ }^{b}$, Ying Zhang ${ }^{b}$, Xu Chen $^{b}$ and K. Kasahara ${ }^{b c}$ \\ ${ }^{a}$ National Astronomical Observatories, Chinese Academy of Sciences, Beijing 100012, China \\ ${ }^{b}$ Key Laboratory of Particle Astrophysics, Institute of High Energy Physics, Chinese Academy of \\ Sciences, Beijing 100049, China \\ ${ }^{c}$ Research Institute for Science and Engineering, Waseda University, Tokyo 169-8555, Japan
}

E-mail: zhailiumingdnao.cas.cn

As the particle lateral density distribution of extensive air showers (EAS) is the key quantity for most of the ground-based cosmic-ray experiments, the lateral distribution function (LDF) of EAS plays an important role in the analysis of the air-shower events. It can be used to derive air-shower size and age, etc, which, in turn, can be used to estimate the energy and particle type of the incident primary cosmic rays. To measure the chemical compositions of primary cosmic rays at the knee energies, the Tibet AS $\gamma$ collaboration started constructing a new hybrid detector system in Tibet, China, in 2014. The new hybrid experiment consists of the air-shower array (Tibet-AS), the air-shower core-detector array (YAC) and the underground water-Cherenkov muon-detector array (MD). Based on a full Monte-Carlo simulation for the new Tibet hybrid experiment (Tibet$\mathrm{AS}+\mathrm{YAC}+\mathrm{MD}$ ), we developed modified LDFs for proton-induced and iron-induced air showers, respectively. As an application of the new LDFs, we derive some characteristic parameters sensitive to the primary composition.

35th International Cosmic Ray Conference - ICRC2017

10-20 July, 2017

Bexco, Busan, Korea

${ }^{*}$ Speaker. 


\section{Introduction}

It is well known that the all-particle energy spectrum of primary cosmic rays follows a broken power law, i.e, the spectrum shape, $\mathrm{dN} / \mathrm{dE} \propto \mathrm{E}^{\gamma}$, steepens at energies around $4 \times 10^{15} \mathrm{eV}$ where the spectral index changes from $\gamma \approx-2.7$ to $\gamma \approx-3.1$ [ [ [ [1]. Such a structure of the all-particle energy spectrum is called the "knee", which is considered to be closely related to the origin of Galactic cosmic rays [ [3]. Many hypotheses and mechanisms have been proposed to explain the knee, e.g [ $[\mathbf{H}$, [], and all these approaches can describe the knee structure well, but there are much discrepancies in the prediction of the individual components at the knee region. Thus, it is important to measure the primary chemical compositions or mass groups at the knee energies, especially, to measure the primary energy spectra of individual component and determine the break energy of spectral index for individual component (Proton, Helium, .... Iron). These measurements will answer whether or not the knee is contributed by the nearby source(s), and will judge whether the knee position of each component relies on either Atomic number (Z) or Mass number (A) or others.

Direct cosmic-ray measurements on board balloons or satellites are the best way to study the chemical composition, while the maximum energy they can cover is up to $10^{14} \mathrm{eV} /$ nucleon at most due to limited detection area or exposure time. We may have no choice but to rely on ground-based air-shower (AS) measurements to study the primary chemical composition around the knee.

To measure the chemical compositions of primary cosmic rays at the knee, the Tibet AS $\gamma$ collaboration has been developing a new hybrid detector system in Tibet, China, since 2014, as described in the next Sec.】.

Since the particle lateral density distribution of extensive air showers (EAS) is the key quantity for most of the ground-based cosmic-ray experiments, the lateral distribution function (LDF) of EAS plays an important role in the analysis of the air-shower events. It can be used to derive air shower size and age, which, in turn, can be used to estimate the energy and particle type of the primary cosmic rays. Based on a full Monte Carlo simulation for the new Tibet hybrid experiment, we analyze differences of proton-induced and iron-induced air showers, and obtain modified LDFs for each of the components.

The new LDFs are applied to derive characteristic parameters which are sensitive to the mass of the primary.

\section{Some details of the new experiment}

The new hybrid experiment consists of three different types of detector arrays: 1) air-shower array (Tibet-AS), 2) Yangbajing air-shower core-detector array (YAC) and 3) underground waterCherenkov muon-detector array (MD) (Fig.W). They are located at Yangbajing (4300 $\mathrm{m}$ a.s.l.; $606 \mathrm{~g} / \mathrm{cm}^{2}$ ) in Tibet, China. The Tibet-AS consists of 789 detector units covering an area of $\sim 50,000 \mathrm{~m}^{2}$; the YAC consists of 124 detector units covering an area of $\sim 500 \mathrm{~m}^{2}$ and the five MD pools consists of 80 cells, with an area of $\sim 4,200 \mathrm{~m}^{2}$. This hybrid experiment is designed not only for observation of air showers of nuclear component origin, but also for observation of

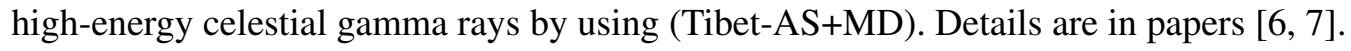

The YAC array is constructed near the center of the Tibet-AS array (Fig.W), and it has been operated simultaneously with Tibet-AS and MD array. For an air-shower event, 1) Tibet-AS pro- 


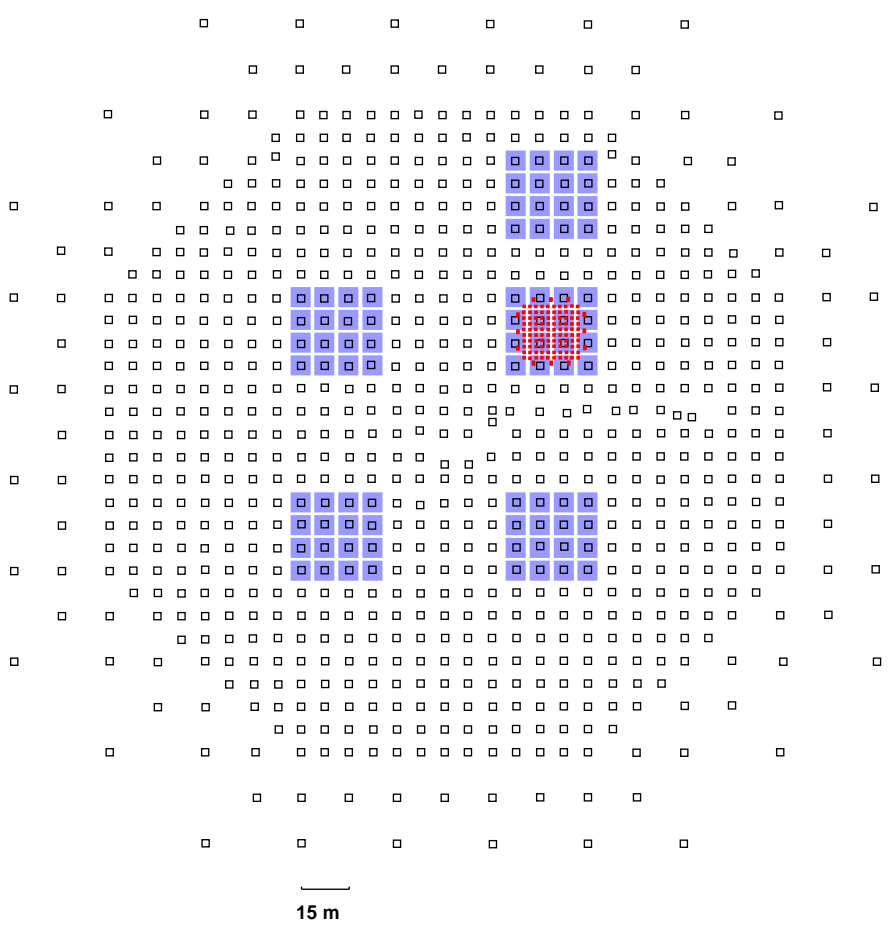

Figure 1: Schematic view of the current new Tibet AS $\gamma$ experiment. Open squares: Tibet-AS consisting of 789 scintillation detectors (each surface area is $0.5 \mathrm{~m}^{2}$ ). Filled blue squares: MD ( 5 pools each of which consists of 16 cells (each $\left.52 \mathrm{~m}^{2}\right)$ ). Filled red squares: YAC consisting of 124 detectors $\left(\right.$ each $0.4 \mathrm{~m}^{2}$ ).

vides the arrival direction $(\theta, \varphi)$ and the air shower size, $N_{e}$ and age $s$ (see later for these) which are interrelated to primary energy; 2) YAC measures mainly high energy electromagnetic particles in the core region so as to obtain the characteristic parameters of air-shower cores; 3) MD is sensitive to high-energy muons above $1 \mathrm{GeV}$. When a YAC event is triggered, its accompanying air shower is simultaneously recorded. The matching between YAC, AS and MD events is made by their arrival time stamps.

\section{Monte Carlo Simulations}

We have carried out a full Monte Carlo (MC) simulation on the development of air showers in the atmosphere using the simulation code Corsika [ [ $]$ ]. Three hadronic interaction models, QGSJET01c, SIBYLL2.1 and EPOS-LHC, are used to generate air-shower events with primary energy above $50 \mathrm{TeV}$. Two primary composition models, namely, "He-poor"and "He-rich" model$\mathrm{s}$ [6, $\mathrm{G}$ ], are used to appraise the systematic errors attributable to primary composition models.

In this simulation, primary cosmic rays within the zenith angles smaller than 60 degrees are thrown into the atmosphere isotropically, and all shower particles in the atmosphere are traced down to the minimum energy of $1 \mathrm{MeV}$. The detector responses to shower particles falling on the detectors of (Tibet-AS+YAC+MD) array are calculated using the Geant4 [Q], where the detector 
performance, trigger efficiency and effective area are adequately taken into account based on the experimental conditions.

\section{Results and Discussion}

\subsection{Lateral distribution function of EAS}
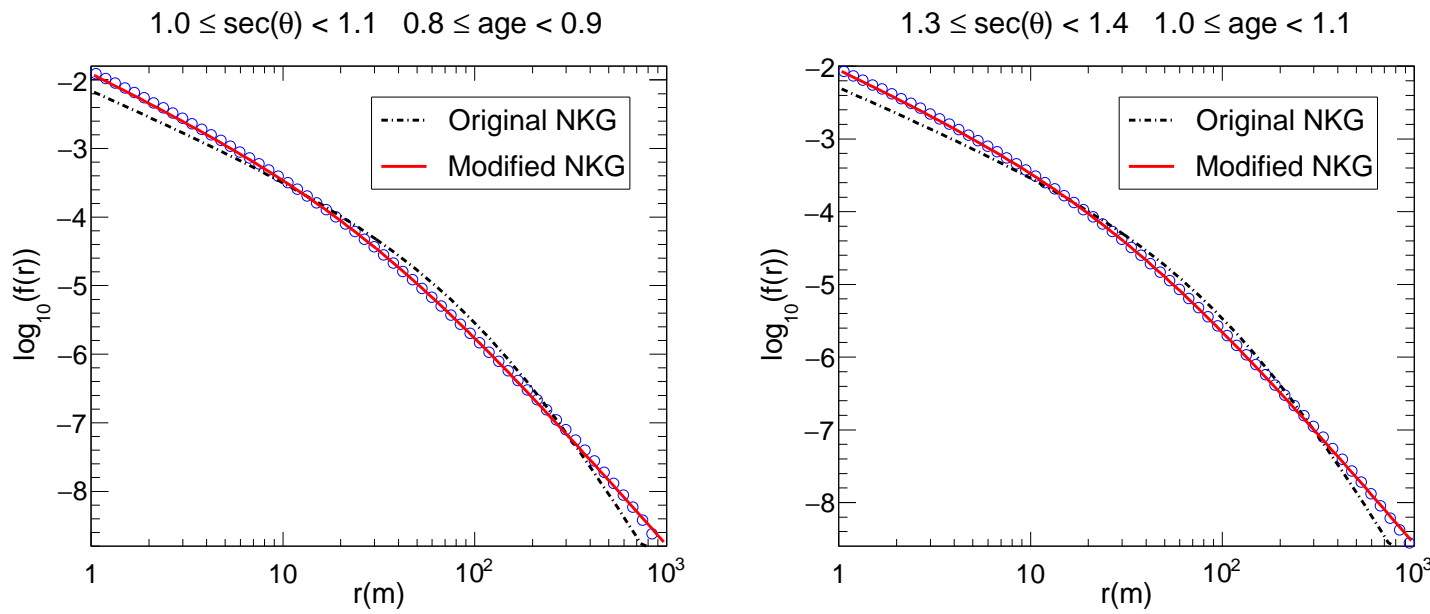

Figure 2: Comparison between the modified NKG function (solid lines) and the original NKG function (dotted lines) in different zenith angles (left: $1.0 \leq \sec (\theta) \leq 1.1$; right: $1.3 \leq \sec (\theta) \leq 1.4$ ). They are fitted to the M.C lateral distribution of energy deposit in the "carpet" array (open circles).

The reconstruction of core position, the arrival direction, the air-shower size $\left(N_{e}\right)$, and the airshower age $(s)$, is done using information of the Tibet-AS array. Here $N_{e}$ is defined as the total effective number of particles in an air shower; it is estimated from the observed energy deposit in each detector of Tibet-AS (in unit of peak position value of the energy deposit distribution due to nearly vertical muons; this is obtained by calibration runs and corresponding M.C with low energy primary particles). The mean error of the core position is estimated as $\sim 5 \mathrm{~m}$, and the mean error of the arrival direction can be estimated as $\sim 0.2^{\circ}$ at energies above $10^{14} \mathrm{eV}$.

In this work, we focus on the lateral distribution function (LDF) of air showers, since the air-shower size $\left(N_{e}\right)$, and the air-shower age $(s)$, in each event are obtained by fitting LDF to experimental data, and they are used to estimate the primary energy and primary particle type.

As the LDF, we use the NKG type function

$$
\begin{aligned}
f(r, s) & =\frac{N_{e}}{C(s)}\left(\frac{r}{r_{m}^{\prime}}\right)^{a(s)}\left(1+\frac{r}{r_{m}^{\prime}}\right)^{b(s)} / r_{m}^{2}, \\
C(s) & =2 \pi B[a(s)+2,-b(s)-a(s)-2] .
\end{aligned}
$$

Here $B$ denotes the beta function. In the original NKG function, $a=s-2, b=s-4.5$ are used and $r_{m}^{\prime}=130 \mathrm{~m}$ at the Yangbajing altitude, but we modify it so that both $a$ and $b$ are bit more complicated functions of age $s$ and we use $r_{m}^{\prime}=30 \mathrm{~m}$ [四. To see the feasibility of two LDFs, age $s$ is determined by fitting $f(r, s)$ to the average MC data for the "carpet" (i.e, completely dense) array 
configuration ${ }^{1}$. The result is shown in Fig.D]; our modified NKG function is a better LDF in this case.

Next, in stead of using "carpet" array, fitting is tried to M.C data for the real 789 Tibet-AS detectors. Fig.3] shows the fractional difference between the true shower size ( $N_{e_{-}}$true, red lines) and the estimated shower size ( $N_{e_{-}}$fit, blue circles): Top figures are obtained by the original NKG function and bottom ones by the modified NKG function, for two different zenith angle regions. While we see $\sim 10 \%$ (for smaller zenith angles) and $\sim 20 \%$ (for larger zenith angles) lower estimate of $N_{e}$ by the original NKG function, the modified NKG function gives almost satisfactory results $(<2 \%)$ as seen in the figures.

Hereafter we use our modified LDF in this paper.
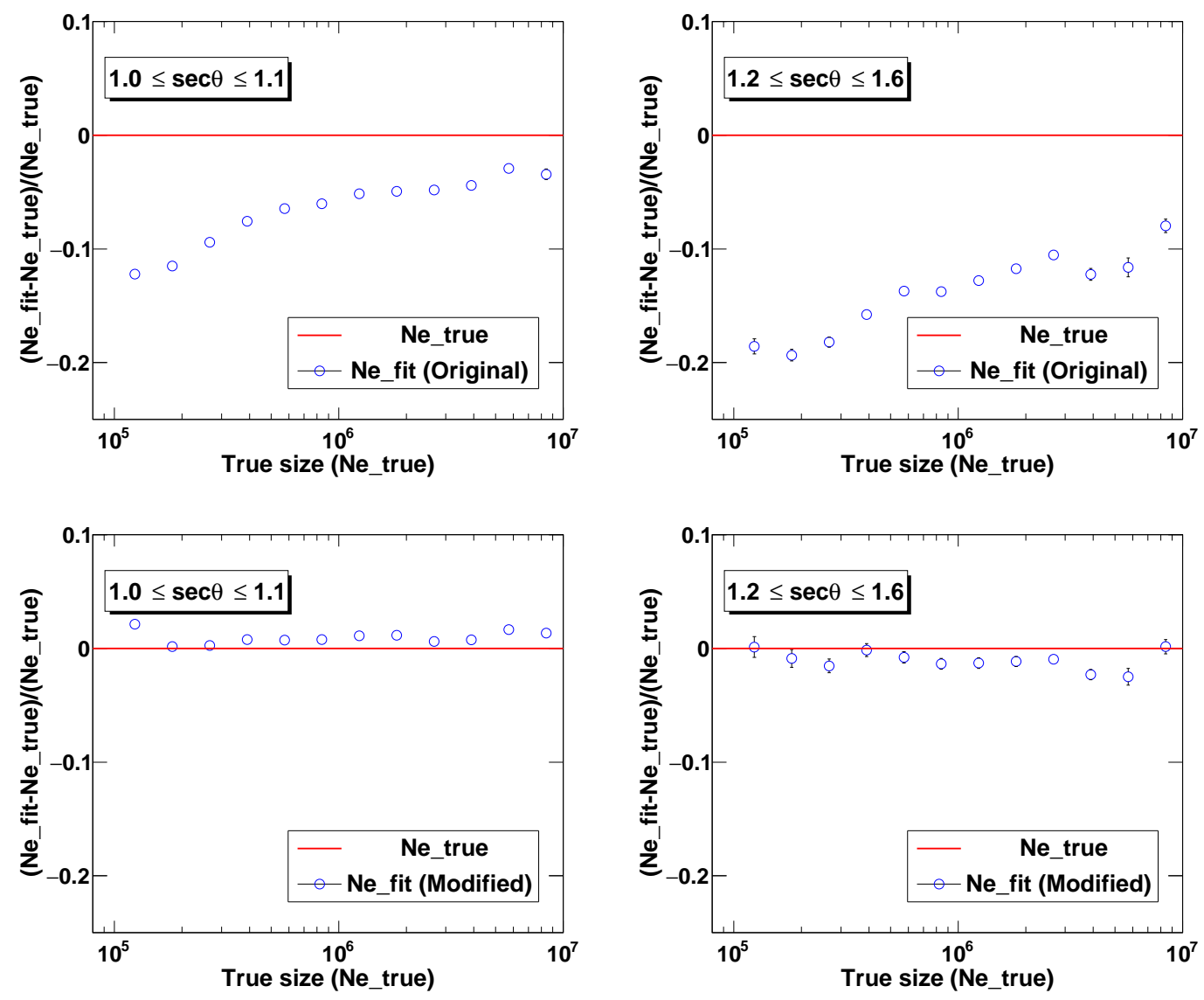

Figure 3: Fractional difference of the estimated shower size ( $N_{e_{-}}$fit, blue circles) from the true shower size ( $N_{e_{-}}$true, red lines): Top one by the original NKG function and bottom by the modified one in different zenith angles.

\subsection{Lateral distribution function for Proton and Iron}

Considering the different feature of AS development for different primary cosmic-ray nuclei,

\footnotetext{
${ }^{1}$ The age, $s$, is originally a parameter expressing the one dimentional development of "electrons" ( $s=1$ corresponds to the shower maximum depth), but we use it as a parameter in the lateral distribution dependent of detector structure, $f(r, s)$. It also reflects the development of A.S but $s=1$ does not mean the shower maximum.
} 
the lateral distribution of air showers for proton (or iron) primaries may be different from that for the all particles, and we try to develop LDFs for proton and iron components, respectively, for the current new Tibet AS $\gamma$ hybrid experiment. This is to get $a(s)$ and $b(s)$ for the case of pure protons and pure irons. Fig.⿴囗十 (left) shows a comparison of $a(s)$ for three different primary compositions: all particles, protons and irons. The right one is the same one for $b(s)$. They are almost the same for the three cases at age $s<1.2$, although there are some differences at $s>1.2$.

Despite the differences at $s>1.2$, it is found that there is little difference between the estimated shower sizes $(\mathrm{Ne})$, and ages $(s)$, whether we use the LDF for protons, irons or all particles. For example, take the iron nuclei primary case, as shown in Fig.[1, the error of estimated $\mathrm{Ne}$ is $\sim 5 \%$ at primary energies around $10^{15} \mathrm{eV}$, independently of the LDFs for the iron or all-particle case.
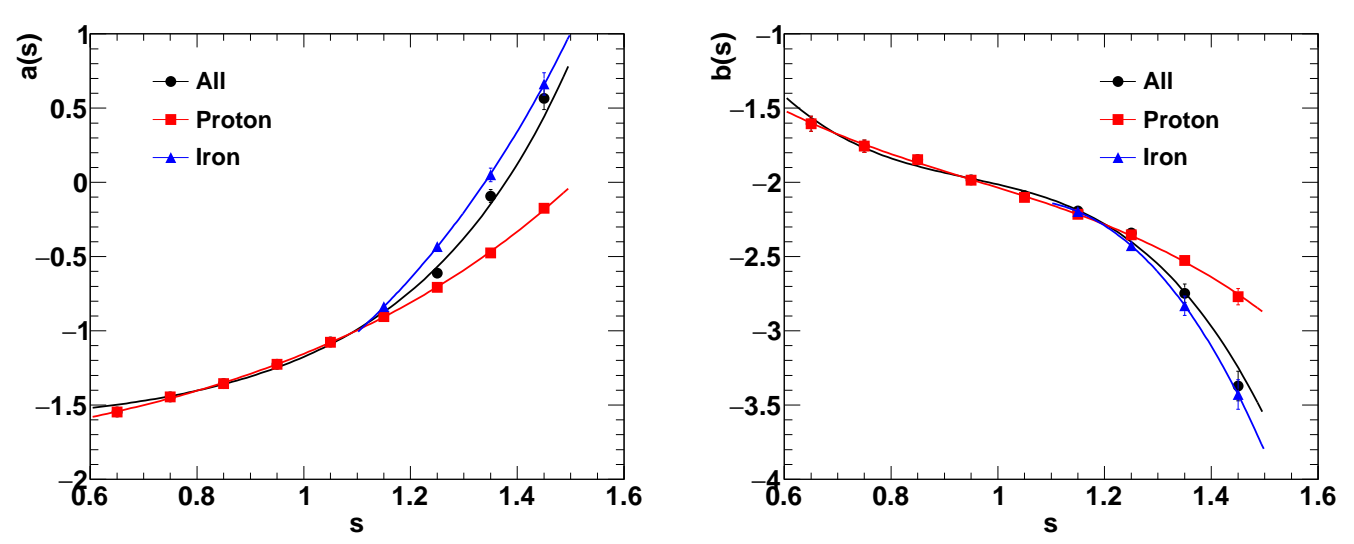

Figure 4: The numerical values of $a$ (left figure) and $b$ (right figure) plotted as a function of age $s$ in the case of pure protons (red squares), pure irons (blue triangles) and all particles (black circles), based on the QGSJET01c+He-poor model, with $1.0 \leq \sec (\theta) \leq 1.1$.

\subsection{Air shower age $(s)$}

When reconstructing the air shower size $(\mathrm{Ne})$, the air shower age $(s)$, is also obtained by fitting the lateral density distribution of air showers. It is sensitive to the primary cosmic-ray type, as shown in Fig.G, which shows $s$ parameter distributions obtained with $a(s)$ and $b(s)$ in the allparticle case. We see different distributions for the proton and iron primary cases.

\subsection{Reconstruction of YAC and MD array}

The reconstruction of air-shower core events is done by the YAC array, and we could obtain the following 5 parameters to characterize an air-shower core event: $N_{h i t}, N_{b}{ }^{t o p}, \Sigma N_{b},\langle R\rangle$ and $\left\langle N_{b} R\right\rangle$, all those parameters are reconstructed from the evaluation of the burst size $\left(N_{b}\right)$ under the lead plate of a YAC detector unit, that is the total energy loss in each scintillator divided by the single peak value. For the MD array, we get the sum of muons $\left(N_{\mu}\right)$ "fired" in the MD array for each air-shower event. Details about the reconstruction of YAC and MD can be seen in paper [ $[\square]$. We did the study of mass composition of primary cosmic rays based on those parameters and the parameters reconstructed by the Tibet-AS array. 


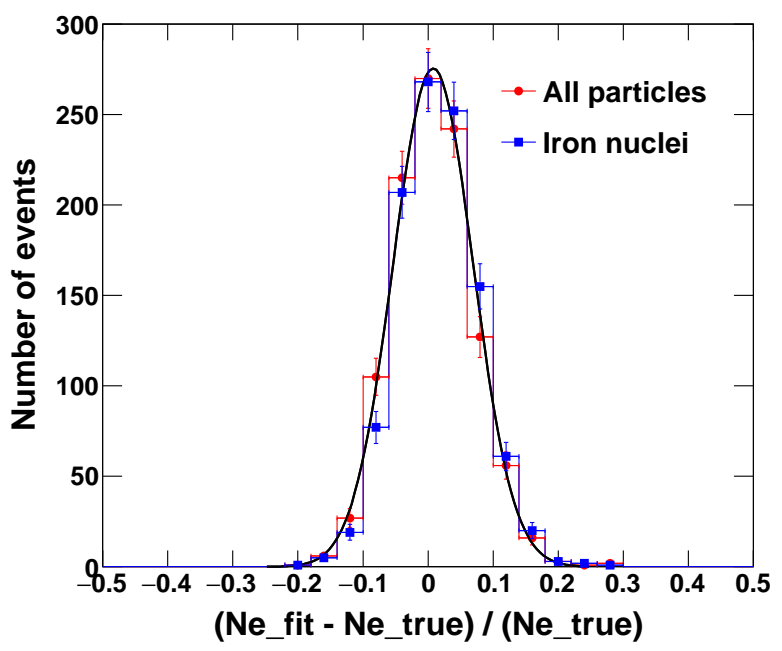

Figure 5: Shower size resolution of iron nuclei is estimated to be $\sim 5 \%$ at primary energy around $10^{15} \mathrm{eV}$. This is independent of whether we use $a(s)$ and $b(s)$ for the all-particle (red circles) or pure iron (blue squares) case (QGSJET01c+He-poor model and $1.0 \leq \sec (\theta) \leq 1.1$ ).
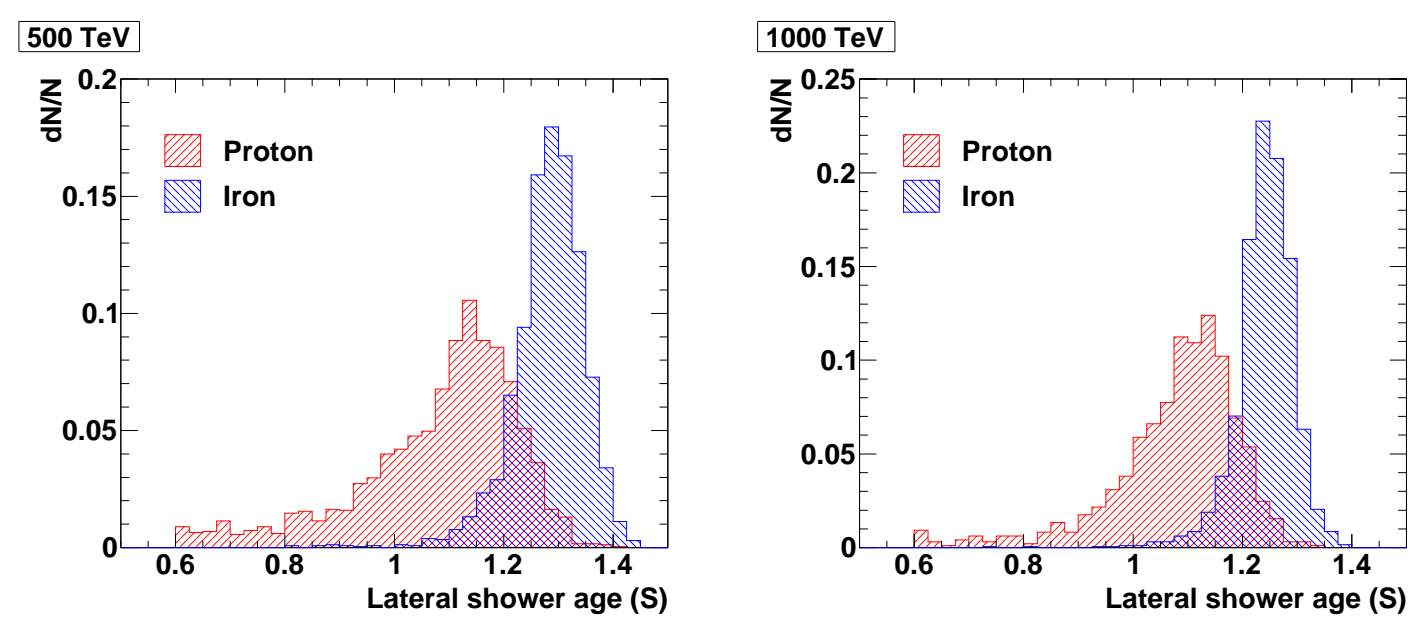

Figure 6: The estimated age parameter $s$ reflects primary type and almost independent of choice of $a(s)$ and $b(s)$. Here we show $s$ distribution with $a(s)$ and $b(s)$ obtained for the all-particle case. Left: primary energy is around $500 \mathrm{TeV}$. Right: primary energy around $1000 \mathrm{TeV}$. In both cases, red is for the proton primary and blue for the iron primary $(1.0 \leq \sec (\theta) \leq 1.1)$.

\section{Summary}

In this paper, we mainly analyzed the differences of proton-induced and iron-induced air shower, and developed the modified LDFs for all particles, proton and iron components, respectively, based on the full Monte Carlo simulation for the new Tibet AS $\gamma$ hybrid experiment. We found that the lateral distribution of shower particles (in terms of energy deposit in detectors) obtained by the Tibet-AS array is well reproduced by the modified NKG function. The shower size $\left(N_{e}\right)$ resolution is estimated to be $\sim 5 \%$ at primary energies around $1000 \mathrm{TeV}$ for iron primaries. The reconstruction 
of characteristic parameters which are sensitive to the mass of the primary, like the air shower age $(s)$, is also discussed here. The results show that the new Tibet hybrid experiment is powerful to study the primary chemical composition, and we look forward to the latest results.

\section{Acknowledgments}

The authors would like to express their thanks to the members of the Tibet AS $\gamma$ collaboration for the fruitful discussion. This work is supported by the National Natural Science Foundation of China (Nos. 11603047, 11533007, 11673041 and 11275212), by the Ministry of Science and Technology of China (No. 2016YFE0125500), by President's International Fellowship Initiative (PIFI), Chinese Academy of Sciences (2017VMA0030), and by the Key Laboratory of Particle Astrophysics, Institute of High Energy Physics, CAS.

\section{References}

[1] M. Amenomori et al., The All-Particle Spectrum of Primary Cosmic Rays in the Wide Energy Range from $10^{14}$ to $10^{17} \mathrm{eV}$ Observed with the Tibet-III Air-Shower Array, Astrophys. J. 678 (2008) 1165

[2] J. R. Hörandel, On the knee in the energy spectrum of cosmic rays, Astropart. Phys. 19 (2003) 193

[3] J. Blümer, R. Engel, and J. R. Hörandel, Cosmic rays from the knee to the highest energies, Prog. Part. Nucl. Phys. 63 (2009) 293

[4] M. Shibata et al., Chemical Composition and Maximum Energy of Galactic Cosmic Rays, Astrophys. J. 716 (2010) 1076

[5] J. R. Hörandel, Models of the knee in the energy spectrum of cosmic rays, Astropart. Phys. 21 (2004) 241 [astro-ph/0402356]

[6] L. M. Zhai et al., Sensitivity of YAC to measure the light-component spectrum of primary cosmic rays at the 'knee' energies, J. Phys. G: Nucl. Part. Phys. 42 (2015) 045201

[7] J. Huang et al., Performance of the Tibet hybrid experiment (YAC-II+Tibet-III+MD) to measure the energy spectra of the light primary cosmic rays at energies 50-10000 TeV, Astropart. Phys. 66 (2015) 18

[8] Heck D. et al., 1998 Forschungszentrum Karlsruhe Report FZKA 6019

[9] Agostinelli S. et al., Geant4 - a simulation toolkit, Nucl. Instrum. Meth. Phys. Res. A 506 (2003) 250 